\title{
Update on the systemic management of radioactive iodine refractory differentiated thyroid cancer (Review)
}

\author{
GEETHU BABU and CESSAL THOMMACHAN KAINICKAL
}

Department of Radiation Oncology, Regional Cancer Center, Medical College Campus, Trivandrum, 695011 Kerala, India

Received June 9, 2020; Accepted September 25, 2020

DOI: $10.3892 / \operatorname{mco} .2020 .2197$

\begin{abstract}
The incidence of differentiated thyroid cancer(DTC) has increased over the last few decades, though it remains to be a rare disease. The prognosis of DTC is excellent; its treatment includes surgery (near-/total thyroidectomy), which is usually followed by remnant thyroid bed ablation using radio-iodine, as well as a risk-stratified follow-ups, including hormone replacement. Treatment of patients who are non-responsive to radioactive iodine (RAI) remains a challenge. Targeted therapies for RAI refractory DTC act primarily through inhibition of cell proliferation, survival and angiogenesis. Tyrosine kinase inhibitors (TKI) have achieved prolonged responses and improved progression-free survival, thereby representing a shift in the treatment of advanced thyroid cancer. There will be number of targeted treatment options for this patient population in the near future. Evidence regarding which drug should be used first and whether there is crossover drug resistance between these drugs is still lacking. Clinicians should be able to choose precisely which patients should be treated with novel targeted therapies after taking into account the following facts: i) TKIs have still not demonstrated a survival benefit. ii) The adverse effects of long-lasting treatment with TKIs could worsen quality of life, which is mostly excellent in these patients before starting treatment with these agents.
\end{abstract}

\section{Contents}

1. Introduction

2. Evolution of treatment

3. Phase 3 trials with sorafenib and lenvatinib

4. Toxicity

5. Discussion

6. Future directions: Ongoing clinical trials

7. Conclusion

Correspondence to: Dr Cessal Thommachan Kainickal, Department of Radiation Oncology, Level 2, Regional Cancer Center, Medical College Campus, Trivandrum, 695011 Kerala, India E-mail: drcessalthomas@gmail.com

Key words: tyrosine kinase inhibitors, thyroid cancer, iodine refractory

\section{Introduction}

The incidence of differentiated thyroid cancer (DTC) has increased over the last few decades, though it remains to be a rare disease (1). The appearance of DTC is dependent on age, sex, family history, radiation exposure and numerous other factors (2). In 2014, approximately 63,000 new cases of DTC were diagnosed compared with only 31,200 new cases in 2009 in the USA. There are $\sim 13,000$ new cases of DTC per year in India (3). The increased usage of diagnostic methods, such as ultrasound of the neck, has led to an increased number of known cases of thyroid cancer, as well as more diagnoses of smaller tumors (4). The prevalence of distant metastases in patients with DTC is very low and metastases tend to affect lung and bone (5).

However, only two thirds of patients with metastases show substantial radioactive iodine (RAI) uptake, even after adequate stimulation with thyroid-stimulating hormone (TSH), and in the absence of excess iodine, only $42 \%$ of them achieve a cure (6). Patients who are non-responsive to RAI are expected to have a life expectancy of 3-5 years, which demonstrates a clear unmet medical need. An expert panel met in September 2012 to propose a working definition and treatment algorithm for these patients (7).

Patients with RAI-refractory DTC fall into four categories, including: i) Patients with metastatic disease who do not take up RAI at the time of initial treatment; ii) patients whose tumors lose the ability to take up RAI in subsequent treatments after previous evidence of uptake. This is often due to the eradication of differentiated cells that take up RAI, but not of poorly differentiated cells that do not take up RAI; iii) patients with multiple large metastases in whom RAI uptake is retained in some lesions but not in others. In such patients, progression is likely to occur in metastases without RAI uptake, in particular when $18 \mathrm{~F}$-fluorodeoxyglucoseuptake is present in these lesions and RAI treatment will not be beneficial in such patients; iv) patients whose disease continues to progress despite substantial uptake of RAI. If progression occurs after a course of adequate radio-iodine treatment, subsequent iodine treatment will be ineffective.

The presence of the aforementioned criteria in a patient does not necessarily mean that the tumor will be unresponsive to iodine treatment, but rather that it predicts the likelihood that the tumor will be unresponsive to iodine treatment. The clinical situation should also be considered before labelling as a radio-iodine refractory disease. 
Loss of thyroid differentiation is one of the hallmarks for radioiodine refractoriness. A hallmark of thyroid dedifferentiation is impairment of $\mathrm{Na} / \mathrm{I}$ symporter (NIS) function. NIS is a plasma membrane glycoprotein located on the basolateral surface of thyroid follicular epithelial cells, and mediates active iodide transport into the cells. Iodine enters the cells via the NIS and emits $\beta$ particles that destroy the follicular cell. Impaired intracellular retention of NIS, or impaired targeting of NIS to the plasma membrane results in NIS loss, producing RAI resistance in a subset of DTCs.

\section{Evolution of treatment}

Improved understanding of oncogenic pathways over the past couple of decades has enabled the development of targeted therapies with very promising efficacy in numerous types of tumor. Targeted therapies for RAI-refractory DTC act primarily through inhibition of cell proliferation, survival and angiogenesis (8). As thyroid cancer becomes more dedifferentiated and aggressive, it accumulates increasingly activating mutations in the PI3 and MAP kinase pathways. Multi-targeted tyrosine kinase inhibitor (TKI) agents utilized for the treatment of RAI refractory DTC have demonstrated response rates ranging between 8 and 49\%, and progression-free survival (PFS) ranging between 10.8 and 18.1 months (9).

Sorafenib is a TKI that inhibits several tyrosine-kinase receptors (TKRs) including $\mathrm{RET}$. It also targets the serine/threonine kinases RAF1 and BRAF. Sorafenib is active against TKRs including vascular endothelial growth factor receptors (VEGFR)2 and 3, platelet derived growth factor receptor (PDGFR)b, c-KIT and Flt3, which are involved in tumor neovascularization and progression. The drug, therefore, may inhibit thyroid cancer growth through antiproliferative and anti-angiogenic mechanisms.

Lenvatinib is an oral, multi-targeted TKI that is active against VEGFRs 1 to 3 (VEGFR1-VEGFR3), fibroblast growth factor receptors 1 to 4 (FGFR1-FGFR4) and PDGFR $\alpha$, which are involved in tumor neovascularization and progression. It is also active against v-kit Hardy-Zuckerman 4 feline sarcoma viral oncogene homolog (KIT) and RET proto-oncogene (RET) signaling pathways, which are implicated in tumor angiogenesis.

Tipifarnib, a selective farnesyl transferase (FTase) inhibitor. Farnesylation of Ras is the rate-limiting step in the post-translational modification of Ras and is required for its oncogenic activity. Tipifarnib induces antiproliferative effects against various human tumor cell lines and has demonstrated clinical activity in several types of malignancy.

Mutations in the PI3K/mTOR/AKT pathway occur in the late stages of thyroid cancer and are associated with aggressive disease. Temsirolimus and Everolimus are inhibitors of mTOR and have demonstrated activity in thyroid cancer.

Targeted agents, in particular the TKIs, hold promise for the treatment of metastatic well-DTC as driver and passenger mutations are increasingly understood. Clinical activity of TKI in thyroid cancer has been demonstrated in phase 2 trials (Table I).

\section{Phase 3 trials with sorafenib and lenvatinib}

Sorafenib trial. DECISION (10) was a multi-center, randomized, double-blind, placebo-controlled, phase 3 trial in which
417 patients from 77 centers in 18 countries were randomized into groups that received sorafenib $400 \mathrm{mg}(\mathrm{n}=207)$ or placebo $(n=210)$ between October 2009 and August 2011. The inclusion criteria for this trial were: i) Locally advanced or metastatic RAI refractory DTC [papillary, follicular (including Hürthle cell)], and poorly differentiated with disease progression within the last 14 months according to Response Evaluation Criteria in Solid Tumors (RECIST) and at least one lesion measurable by CT or MRI scans according to RECIST; ii) adequate bone marrow, liver and renal function, and a serum thyroid-stimulating hormone concentration $<0.5 \mathrm{mIU} / 1$.

The primary endpoint was PFS, assessed every 8 weeks. Secondary endpoints were overall survival (OS), time to progression, objective response rate, complete or partial response, disease control rate and stable disease, and duration of response. Response and progression were assessed with repeat CT or MRI scans, performed $\geq 4$ weeks after starting treatment. The National Cancer Institute Common Terminology Criteria for Adverse Events version 3.0 was used to assess safety.

The median time from randomization until last known follow-up was 16.2 months (range 0.03-33.20 months). Significant improvement in median PFS was observed for sorafenib compared with placebo [10.8 vs. 5.8 months; hazard ratio, 0.59; 95\% confidence interval (CI) 0.45-0.76; $\mathrm{P}<0.0001]$, with a $41 \%$ decrease in the risk of progression or death. An exploratory subgroup analysis of PFS showed consistent improvement in all prespecified subgroups. The objective response rate was $12.2 \%(24 / 196)$ in the sorafenib compared with $0.5 \%(1 / 201)$ in the placebo group $(\mathrm{P}<0.0001)$. At the time of analysis, the median overall survival hadnot been reached, potentiallyaffected by the fact that the majority of patients from the placebo group hadalready or will move over to the sorafenib group.

Lenvatinib trial. The lenvatinib phase 3 trial (11) was a randomized, double-blind, placebo controlled, multi-center study, between August 5, 2011 and October 4, 2012 in which 392 eligible patients were randomly assigned in a 2:1 ratio to receive oral lenvatinib at a dose of $24 \mathrm{mg}$ once daily (261 patients) or placebo in 28-day cycles (131 patients). The inclusion criteria for this trial were: i) Histologically and/or cytologically confirmed diagnosis of RAI-refractory showing progression within 12 months; ii) eligible patients had received no prior therapy with a TKI or had received one prior treatment regimen with a TKI.

Patients were stratified according to age, geographic region and receipt or non-receipt of prior TKI treatment. All patients in the trial received treatment and were included in the efficacy and safety analyses. The baseline characteristics of the patients were similar in the two groups.

The primary endpoint was PFS, and the secondary endpoints were the response rate defined as the best objective response (complete or partial) according to RECIST version 1.1 and OS. Adverse effects were assessed according to the National Cancer Institute Common Terminology Criteria for Adverse Events version 4.0.

The median duration of follow-up was 17.1 months in the lenvatinib group, and 17.4 months in the placebo group. At the time of data cut-off (November 15, 2013), 130 patients were 


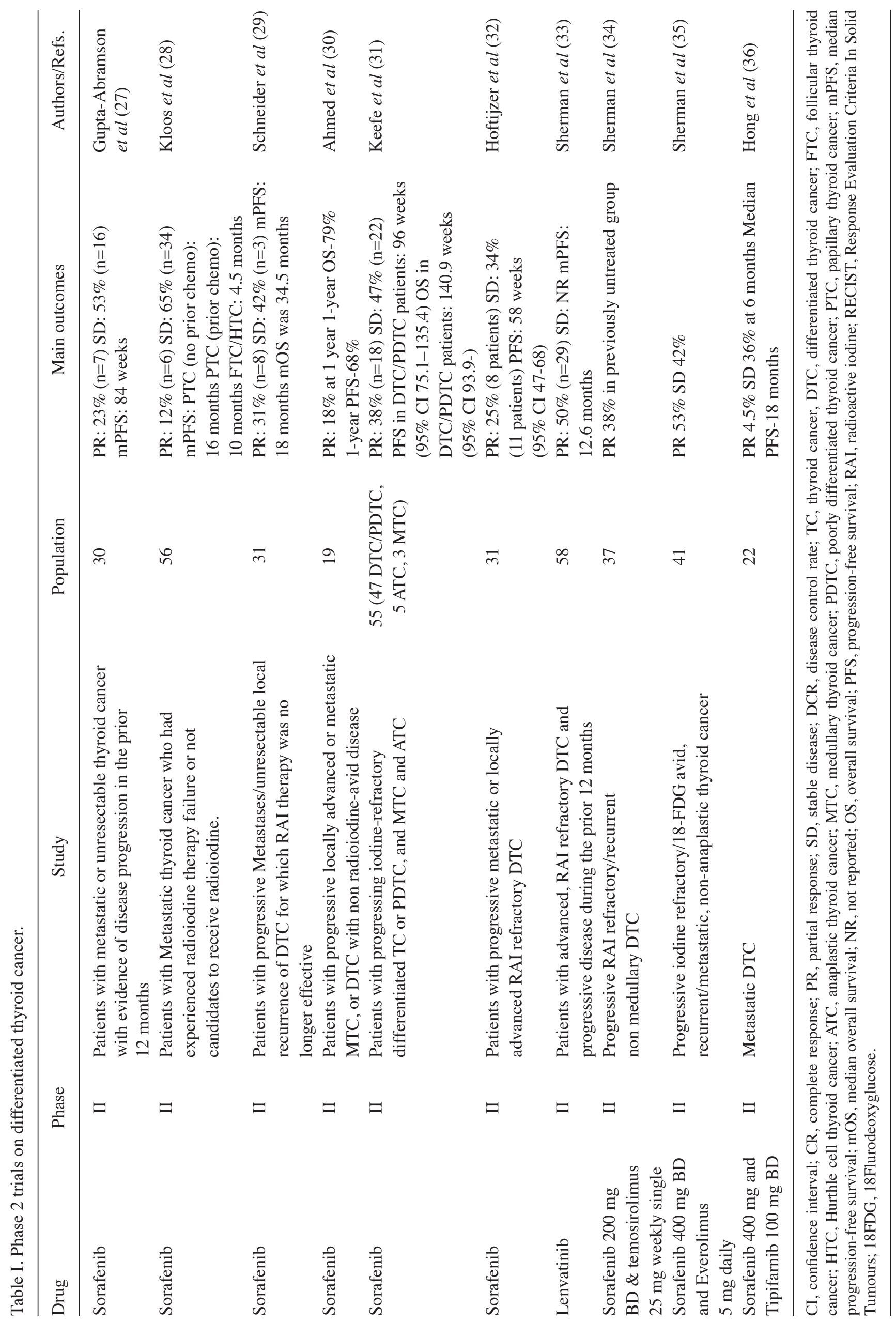


still continuing to receive blinded treatment [122 patients in the lenvatinib arm (46.7\%) and 8 patients assigned to placebo (6.1\%)]. Of the 114 eligible patients, 109 (95.6\%) who received placebo and had tumor progression elected to receive lenvatinib. Of the patients assigned to lenvatinib, 41 (15.7\%) subsequently received additional anticancer therapies for disease progression.

The median PFS was improved with lenvatinib compared with placebo (18.3 vs. 3.6 months; hazard ratio for progression or death, 0.21; 99\% CI, 0.14-0.31; P<0.001). PFS benefit associated with lenvatinib was observed in all prespecified subgroups. The 6-month PFS rates were $77.5 \%$ in the lenvatinib group and $25.4 \%$ in the placebo group. The response rate was higher in the lenvatinib group (64.8 vs. $5 \%$; $\mathrm{P}<0.001)$. The median OS was not reached in either group.

\section{Toxicity}

In the Decision trial on sorafenib, adverse events occurred in 204 out of 207 (98.6\%) patients receiving sorafenib and in 183 of 209 (87.6\%) patients receiving placebo. These events were primarily grades 1 or 2 and tended to occur early in treatment. The most common adverse events in the sorafenib group were hand-foot skin reaction (HFSR), rash or desquamation, fatigue, weight loss diarrhea, alopecia and hypertension. An increase in serum thyroid-stimulating hormone concentration $>0.5 \mathrm{mIU} / 1$ was recorded as an adverse event in $33.3 \%(69 / 207)$ of patients in the sorafenib group, and hypocalcemia in 18.8\% (39/207). Dose interruptions, reductions or withdrawals due to adverse events occurred in 66.2\% (137/207), 64.3\% (133/207), and $18.8 \%(39 / 207)$, respectively, of patients receiving sorafenib, and in $25.8 \%(54 / 209), 9.1 \%(19 / 209)$ and $3.8 \%(8 / 209)$, respectively, of patients receiving placebo. The most common reason for sorafenib dose interruptions, reductions and withdrawals was HFSR. Serious adverse events occurred in 77 (37.2\%) patients receiving sorafenib and 55 (26.3\%) receiving placebo. There were 12 deaths in the sorafenib group, and six in the placebo group. In the sorafenib group, seven deaths were due to underlying disease, two due to unknown causes, and one each due to lung infection, chronic obstructive lung disease and myocardial infarction.

In the Lenvatinib trial, the incidence of treatmentassociated adverse effects (of all grades) was $97.3 \%$ in the lenvatinib group, and $59.5 \%$ in the placebo group, and the incidence of grade 3 or higher adverse effects was $75.9 \%$ in the lenvatinib group, and $9.9 \%$ in the placebo group. Adverse effects that developed in the lenvatinib group were hypertension, proteinuria, arterial thromboembolic effects, venous thromboembolic effects, renal failure, including acute renal failure, hepatic failure, gastrointestinal fistula, corrected QT prolongation and posterior reversible encephalopathy syndrome. Out of the total 118 deaths that occurred, 71 were in the lenvatinib group (27.2\%) and 47 were in the placebo group $(35.9 \%)(\mathrm{P}=0.08)$. The majority of deaths were due to disease progression. Of these, 6 deaths $(2.3 \%)$ were considered by the investigator to be treatment-related, including 1 case each of pulmonary embolism, hemorrhagic stroke, and general deterioration of physical health; 3 cases were reported as not otherwise specified deaths, or sudden deaths.

\section{Discussion}

The multikinase inhibitors have achieved prolonged responses and improved PFS, and they represent a shift in the treatment of advanced thyroid cancer. Sorafenib and lenvatinib are being assessed for the treatment of advanced RAI-refractory DTC in phase 3 clinical trials, with positive results emerging already from the sorafenib and lenvatinib trial. Although the SELECT and DECISION trials tested TKIs for the treatment of advanced RAI-refractory DTC, there are slight but marked differences between these two trials, and these differences should be taken into account when interpreting the data. Differences between the two trials were: i) Previous therapy was not allowed in the DECISION trial but was permitted in the SELECT trial, including one previous treatment with a VEGFR inhibitor; ii) centrally confirmed progressive disease was the inclusion criteria in the SELECT trial, whereas in the DECISION trial, disease progression was assessed by the investigators; iii) the disease progression treatment offered differed in the two trials; iv) a higher number of patients had crossed over between the two treatment groups in the SELECT trial; v) sex race, geographic region, ECOG Performance status, time from diagnosis, histology and site of metastases differed within and between trials. These differences could influence response rates and PFS, making it difficult to compare data between the trials.

Inhibition of unique targets, including FGFRs, is a distinguishing feature of lenvatinib (12). FGF/FGFR is one of the important molecular drivers of tumor growth in the pathogenesis of thyroid cancer. Elevated expression levels of FGF2, FGFR1, FGFR3 and FGFR4 have been detected in human thyroid carcinoma compared with normal thyroid tissue (13). The FGF/FGFR pathway is part of an escape mechanism to VEGF-targeted anti-angiogenic therapies (14). Elevation of FGF23 levels has been demonstratedto be a surrogate marker of FGFR1 inhibition (15).

Pharmacodynamic biomarkers for the inhibition of VEGF and FGF signaling pathways are changes in baseline levels of VEGF and FGF23 (15). In the lenvatinib arm of the select trial, along with VEGF levels, FGF23 levels increased consistently, which is suggestive of lenvatinib-mediated VEGFR and FGFR target engagement and signaling inhibition. To the best of our knowledge, this is the first report of a TKI-mediated increase in FGF23 levels in a phase 3 study. There was an observed PFS improvement in patients, with greater increases in FGF23 levels while receiving lenvatinib, which is suggestive of the essential role of FGFR inhibition in patients with radio-iodine refractory DTC (16). Lenvatinib has a direct oncogenic effect on controlling tumor cell proliferation by inhibiting RET, c-KIT and PDGFR $\beta$, and also affects the tumor microenvironment by blocking FGFR and PDGFR $\beta$ (17).

\section{Future directions: Ongoing clinical trials}

The multikinase inhibitors have achieved prolonged responses and improved PFS. In the near future, a number of treatment options for this group of patients will be available, but with little evidence regarding which drug to be used first and whether or not there is crossover of drug resistance. Furthermore, in this group of patients, chance of cure is less likely and TKIs 
still have not demonstrated a survival benefit, and the adverse effects of long-lasting treatment with TKIs could worsen quality of life. It should be possible to precisely select which patients should be treated with novel targeted therapies by taking these factors into account. Access and adherence to close monitoring with continued assessment of adverse effects and quality of life should be considered in the decision to start therapy. Table II presents ongoing clinical trials.

Specific molecular profiles (18) are more likely to result in RAI-refractory disease (19). The majority of patients have asymptomatic, slowly progressive disease, even in the presence of distant metastases. Patients with RAI-refractory disease exhibiting symptoms confined to a single site benefit from local treatments such as surgery, external beam radiotherapy, or thermal ablation, depending on the site of the lesion and local expertise. When disease progression occurs at multiple sites in patients with target lesions $>1-2 \mathrm{~cm}$ in diameter, treatment with TKIs should be considered (20); however, TKIs have side effects (21). Greater experience in their use and improved knowledge of the risk factors for these adverse effects (22) are likely to improve their tolerance.

For BRAF- or ALK-mutant tumors, specific inhibitors may be used (7). Drugs capable of restoring RAI uptake have attracted great interest. Selumetinib (23) (a selective MEK inhibitor) and dabrafenib (24) (a selective BRAF inhibitor used in BRAF-mutated tumors) have been shown to increase RAI uptake by RAI-refractory tumor tissues. Immunotherapy also appears to be a promising approach to thyroid cancer, alone or in combination with other drugs. These agents have yet to be approved.

Vandetanib was tested in a phase 2 trial that was performed in 145 patients with advanced DTC. This trial demonstrated longer PFS for vandetanib (median PFS, 11.1 months) than those in the placebo group (5.9 months) (hazard ratio, 0.63) (25).

Currently, a double-blind, placebo-controlled phase 3 study (VERIFY, NCT01876784) is evaluating the efficacy of vandetanib in advanced, radio iodine-refractory DTC. The most common adverse events for vandetanib in all trials were clinically insignificant QT interval prolongation, diarrhea, rash, nausea, hypertension, asthenia and fatigue. In patients with progressive metastatic disease, enrolment in clinical trials should be considered and encouraged in order to improve both clinical case outcomes and medical knowledge in the field (26).

\section{Conclusion}

For patients with RAI-refractory DTC, a disease for which there were previously few treatment options available, the advent of targeted systemic therapies represents a major advance. However, the best way to use these targeted treatments in DTC, particularly in determining who to treat and when to start treatment with TKIs, is still under investigation. TKIs are usually administered for longer duration, and adverse effects associated with TKI have a great impact on quality of life and this should be taken into consideration by the clinician prior to treatment initiation. It will be essential to maintain an awareness of the differences in responses and adverse effect profiles of each agent as more new systemic agents become available. As a result, management of each patient may differ from one to another, at least to a certain extent, for each targeted agent.

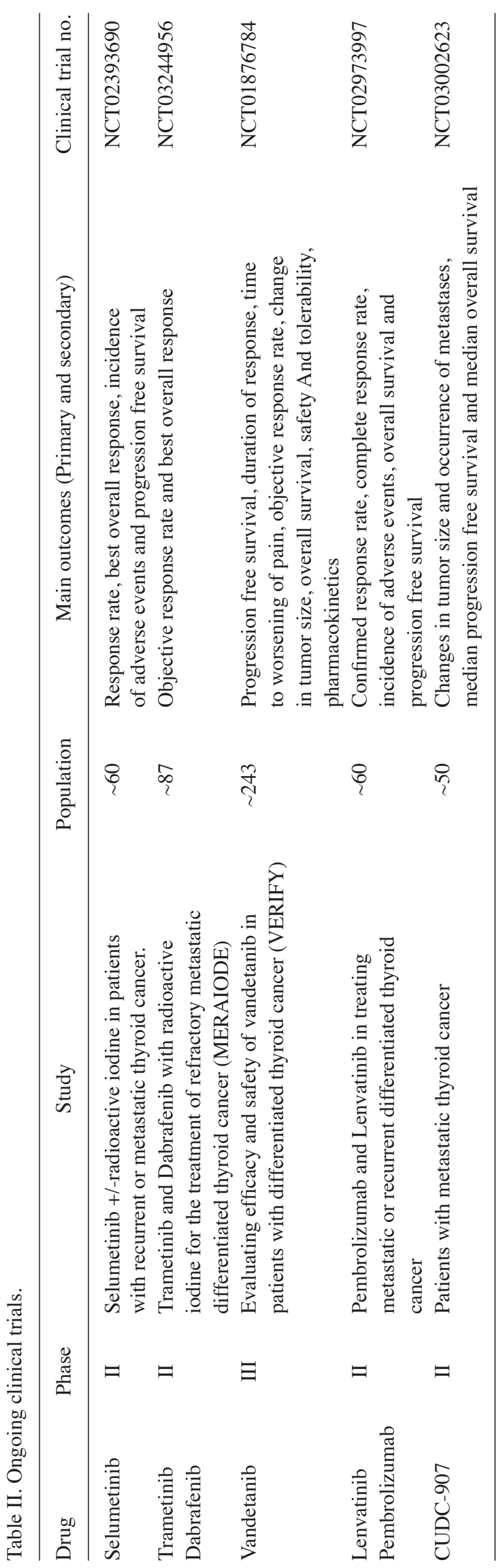




\section{Acknowledgements}

Not applicable.

\section{Funding}

Not funding was received.

\section{Availability of data and materials}

Not applicable.

\section{Authors' contributions}

GB performed the literature search, wrote the manuscript, prepared the tables and edited the manuscript. CTK designed the study, performed literature searches, wrote and edited the manuscript. All authors read and approved the final manuscript.

\section{Ethics approval and consent to participate}

Not applicable.

\section{Patient consent for publication}

Not applicable.

\section{Competing interests}

The authors declare that they have no competing interests.

\section{References}

1. Alonso-Gordoa T, Díez JJ, Durán M and Grande E: Advances in thyroid cancer treatment: Latest evidence and clinical potential. Ther Adv Med Oncol 7: 22-38, 2015.

2. Hegedüs L: Clinical practice. The thyroid nodule. N Engl J Med 351: 1764-1771, 2004

3. GLOBOCAN 2012 [Internet]. Available from: http://globocan. iarc.fr/old/summary_table_pop-html.asp?selection=89356\&title= India \&sex $=0 \&$ type $=0 \&$ window $=1 \&$ sort $=0 \&$ submit $=\% \mathrm{C} 2 \% \mathrm{~A} 0$ Execute\%C2\%A0. Accessed September 10, 2018.

4. Leenhardt L, Bernier MO, Boin-Pineau MH, Conte Devolx B, Maréchaud R, Niccoli-Sire P, Nocaudie M, Orgiazzi J, Schlumberger M, Wémeau JL, et al: Advances in diagnostic practices affect thyroid cancer incidence in France. Eur J Endocrinol 150: 133-139, 2004.

5. BenbassatCA, Mechlis-Frish S and Hirsch D: Clinicopathological characteristics and long-term outcome in patients with distant metastases from differentiated thyroid cancer. World J Surg 30: 1088-1095, 2006.

6. Durante C, Haddy N, Baudin E, Leboulleux S, Hartl D, Travagli JP, Caillou B, Ricard M, Lumbroso JD, De Vathaire F and Schlumberger M: Long-term outcome of 444 patients with distant metastases from papillary and follicular thyroid carcinoma: Benefits and limits of radioiodine therapy. Clin Endocrinol Metab 91: 2892-2899, 2006.

7. Cabanillas ME, Patel A, Danysh BP, Dadu R, Kopetz S and Falchook G: BRAF Inhibitors: Experience in thyroid cancer and general review of toxicity. Horm Cancer 6: 21-36, 2015.

8. Worden F: Treatment strategies for radioactive iodine-refractory differentiated thyroid cancer. Ther Adv Med Oncol 6: 267-279, 2014.

9. Cabanillas ME, Schlumberger M, Jarzab B, Martins RG, Pacini F, Robinson B, McCaffrey JC, Shah MH, Bodenner DL, Topliss D, et al: A phase 2 trial of lenvatinib (E7080) in advanced, progressive, radioiodine-refractory, differentiated thyroid cancer: A clinical outcomes and biomarker assessment. Cancer 121: 2749-2756, 2015
10. Brose MS, Nutting CM, Jarzab B, Elisei R, Siena S, Bastholt L, de laFouchardiere C,PaciniF,Paschke R, Shong YK, et al: Sorafenib in radioactive iodine-refractory, locally advanced or metastatic differentiated thyroid cancer: A randomised, double-blind, phase 3 trial. Lancet 384: 319-328, 2014.

11. Schlumberger M, Tahara M, Wirth LJ, Robinson B, Brose MS, Elisei R, Habra MA, Newbold K, Shah MH, Hoff AO, et al: Lenvatinib versus placebo in radioiodine-refractory thyroid cancer. N Engl J Med 372: 621-630, 2015.

12. St Bernard R, Zheng L, Liu W, Winer D, Asa SL and Ezzat S: Fibroblast growth factor receptors as molecular targets in thyroid carcinoma. Endocrinology 146: 1145-1153, 2005.

13. Boelaert K, McCabe CJ, Tannahill LA, Gittoes NJL, Holder RL, Watkinson JC, Bradwell AR, Sheppard MC and Franklyn JA: Pituitary tumor transforming gene and fibroblast growth factor-2 expression: Potential prognostic indicators in differentiated thyroid cancer. J Clin Endocrinol Metab 88: 2341-2347, 2003.

14. Bergers $G$ and Hanahan D: Modes of resistance to anti-angiogenic therapy. Nat Rev Cancer 8: 592-603, 2008.

15. Kim KB, Chesney J, Robinson D, Gardner H, Shi MM and Kirkwood JM: Phase I/II and pharmacodynamic study of dovitinib (TKI258), an inhibitor of fibroblast growth factor receptors and VEGF receptors, in patients with advanced melanoma. Clin Cancer Res 17: 7451-7461, 2011.

16. Tahara M, Schlumberger M, Elisei R, Habra MA, Kiyota N, Paschke R, Dutcus CE, Hihara T, McGrath S, Matijevic M, et al: Exploratory analysis of biomarkers associated with clinical outcomes from the study of lenvatinib in differentiated cancer of the thyroid. Eur J Cancer 75: 213-221, 2017.

17. Stjepanovic N and Capdevila J: Multikinase inhibitors in the treatment of thyroid cancer: Specific role of lenvatinib. Biologics 8: 129-139, 2014.

18. Xing M, Westra WH, Tufano RP, Cohen Y, Rosenbaum E, Rhoden KJ, Carson KA, Vasko V, Larin A, Tallini G, et al: BRAF mutation predicts a poorer clinical prognosis for papillary thyroid cancer. J Clin Endocrinol Metab 90: 6373-6379, 2005.

19. Durante C, Puxeddu E, Ferretti E, Morisi R, Moretti S, Bruno R, Barbi F, Avenia N, Scipioni A, Verrienti A, et al: BRAF mutations in papillary thyroid carcinomas inhibit genes involved in iodine metabolism. J Clin Endocrinol Metab 92: 2840-2843, 2007.

20. Cabanillas ME, McFadden DG and Durante C: Thyroid cancer. Lancet 388: 2783-2795, 2016.

21. Lamartina L, Ippolito S, Danis M, Bidault F, Borget I, Berdelou A, Al Ghuzlan A, Hartl D, Blanchard P, Terroir M, et al: Antiangiogenic tyrosine kinase inhibitors: Occurrence and risk factors of hemoptysis in refractory thyroid cancer. J Clin Endocrinol Metab 101: 2733-2741, 2016.

22. Blevins DP, Dadu R, Hu M, Baik C, Balachandran D, Ross W, Gunn B and Cabanillas ME: Aerodigestive fistula formation as a rare side effect of antiangiogenic tyrosine kinase inhibitor therapy for thyroid cancer. Thyroid 24: 918-922, 2014.

23. Ho AL, Grewal RK, Leboeuf R, Sherman EJ, Pfister DG, Deandreis D, Pentlow KS, Zanzonico PB, Haque S, Gavane S, et al: Selumetinib-enhanced radioiodine uptake in advanced thyroid cancer. N Engl J Med 368: 623-632, 2013.

24. Rothenberg SM, McFadden DG, Palmer EL, Daniels GH and Wirth LJ: Redifferentiation of iodine-refractory BRAF V600E-mutant metastatic papillary thyroid cancer with dabrafenib. Clin Cancer Res 21: 1028-1035, 2015.

25. Leboulleux S, Bastholt L, Krause T, de la Fouchardiere C, Tennvall J, Awada A, Gómez JM, Bonichon F, Leenhardt L, Soufflet C, et al: Vandetanib in locally advanced or metastatic differentiated thyroid cancer: A randomised, double-blind, phase 2 trial. Lancet Oncol 13: 897-905, 2012.

26. Bible KC, Cote GJ, Demeure MJ, Elisei R, Jhiang S and Ringel MD; International Thyroid Oncology Group Correlative Science Committee: Correlative studies in clinical trials: A position statement from the international thyroid oncology group. J Clin Endocrinol Metab 100: 4387-4395, 2015.

27. Gupta-Abramson V, Troxel AB, Nellore A, Puttaswamy K, Redlinger M, Ransone K, Mandel SJ, Flaherty KT, Loevner LA, O'Dwyer PJ and Brose MS: Phase II trial of sorafenib in advanced thyroid cancer. J Clin Oncol 26: 4714-4719, 2008.

28. Kloos RT, Ringel MD, Knopp MV, Hall NC, King M, Stevens R, Liang J, Wakely PE Jr, Vasko VV, Saji M, et al: Phase II trial of sorafenib in metastatic thyroid cancer. J Clin Oncol 27: 1675-1684, 2009. 
29. Schneider TC, Abdulrahman RM, Corssmit EP, Morreau $H$ Smit JW and Kapiteijn E: Long-term analysis of the efficacy and tolerability of sorafenib in advanced radio-iodine refractory differentiated thyroid carcinoma: Final results of a phase II trial. Eur J Endocrinol 167: 643-650, 2012.

30. Ahmed M, Barbachano Y, Riddell A, Hickey J, Newbold KL, Viros A, Harrington KJ, Marais R and Nutting CM: Analysis of the efficacy and toxicity of sorafenib in thyroid cancer: A phase II study in a UK based population. Eur J Endocrinol 165: 315-322, 2011

31. Keefe SM, Troxel AB, Rhee S, Puttaswamy K, O'Dwyer PJ, Loevner LA, Mandel J and Brose MS: Phase II trial of sorafenib in patients with advanced thyroid cancer. J Clin Oncol 29 (Suppl 15): S5562, 2011.

32. Hoftijzer H, Heemstra KA, Morreau H, Stokkel MP, Corssmit EP Gelderblom H, Weijers K, Pereira AM, Huijberts M, Kapiteijn E, et al: Beneficial effects of sorafenib on tumor progression, but not on radioiodine uptake, in patients with differentiated thyroid carcinoma. Eur J Endocrinol 161: 923-931, 2009.

33. Sherman SI, Jarzab B, Cabanillas ME, Licitra LF, Pacini F, Martins R, Robinson B, Ball D, McCaffrey J, Shah MH, et al: A phase II trial of the multitargeted kinase inhibitor E7080 in advanced radioiodine (RAI)-refractory differentiated thyroid cancer (DTC). J Clin Oncol 29 (15 Suppl): S5503, 2011.
34. Sherman EJ, Ho AL, Fury MG, Baxi SS, Haque S, Korte SH, Smith-Marrone S, Xiao H, Ghossein RA, Fagin JA, et al: A phase II study of temsirolimus/sorafenib in patients with radioactive iodine (RAI)-refractory thyroid carcinoma. J Clin Oncol 30 (15 Suppl): S5514, 2012.

35. Sherman EJ, Ho AL, Fury MG, Baxi SS, Haque S, Lipson BL, Kurz S, Fagin JA and Pfister DG: Phase II study of everolimus and sorafenib for the treatment of metastatic thyroid cancer. J Clin Oncol 31 (Suppl 15): S6024, 2013.

36. Hong DS, Cabanillas ME, Wheler J, Naing A, Tsimberidou AM, Ye L, Busaidy NL, Waguespack SG, Hernandez M, El Naggar AK, et al: Inhibition of the Ras/Raf/MEK/ERK and RET kinase pathways with the combination of the multikinase inhibitor sorafenib and the farnesyltransferase inhibitor tipifarnib in medullary and differentiated thyroid malignancies. J Clin Endocrinol Metab 96: 997-1005, 2011. 